University of Nebraska - Lincoln

DigitalCommons@University of Nebraska - Lincoln

2020

\title{
Attosecond-pulse metrology based on high-order harmonic generation
}

T. S. Sarantseva

M. V. Frolov

N. L. Manakov

A. A. Silaev

A. A. Romanov

See next page for additional authors

Follow this and additional works at: https://digitalcommons.unl.edu/physicsstarace

Part of the Atomic, Molecular and Optical Physics Commons, Elementary Particles and Fields and String Theory Commons, and the Plasma and Beam Physics Commons

This Article is brought to you for free and open access by the Research Papers in Physics and Astronomy at DigitalCommons@University of Nebraska - Lincoln. It has been accepted for inclusion in Anthony F. Starace Publications by an authorized administrator of DigitalCommons@University of Nebraska - Lincoln. 
Authors

T. S. Sarantseva, M. V. Frolov, N. L. Manakov, A. A. Silaev, A. A. Romanov, N. V. Vvedenskii, and Anthony F. Starace 


\title{
Attosecond-pulse metrology based on high-order harmonic generation
}

\author{
T. S. Sarantseva, ${ }^{1,2}$ M. V. Frolov $\odot,{ }^{1}$ N. L. Manakov $\odot,{ }^{1}$ A. A. Silaev $\odot,{ }^{1,2,3}$ A. A. Romanov, ${ }^{1,2,3}$ \\ N. V. Vvedenskii ${ }^{\circledR},{ }^{1,2,3}$ and Anthony F. Starace ${ }^{4, *}$ \\ ${ }^{1}$ Department of Physics, Voronezh State University, Voronezh 394018, Russia \\ ${ }^{2}$ Institute of Applied Physics, Russian Academy of Sciences, Nizhny Novgorod 603950, Russia \\ ${ }^{3}$ Department of Radiophysics, University of Nizhny Novgorod, Nizhny Novgorod 603950, Russia \\ ${ }^{4}$ Department of Physics and Astronomy, University of Nebraska, Lincoln, Nebraska 68588-0299, USA
}

(Received 13 September 2019; published 3 January 2020)

\begin{abstract}
An all-optical method to retrieve the temporal intensity profile of an extreme ultraviolet (XUV) attosecond pulse is proposed based on XUV-assisted high-order harmonic generation (HHG) by an intense infrared (IR) pulse. For a harmonic located on the XUV-induced high-energy plateau (beyond the IR HHG plateau), the measured harmonic yield as a function of the time delay between the XUV and IR pulses is shown to accurately map the temporal intensity profile of the XUV pulse. Single-color and two-color orthogonal, linearly polarized IR pulses are used to demonstrate the method.
\end{abstract}

DOI: 10.1103/PhysRevA.101.013402

\section{INTRODUCTION}

Observations of ultrafast electron dynamics on few-femtosecond and attosecond time scales have become accessible by means of pump-probe experiments with isolated attosecond pulses (IAPs) [1-6]. There are two available sources for IAPs: IAPs based on high-order harmonic generation (HHG) [with relatively small outcome pulse energy and duration of tens of attoseconds] [7,8] and IAPs based on free-electron lasers (FELs) [with higher outcome pulse energy and duration up to hundreds of attoseconds] $[9,10]$. It should be emphasized that a great advance of HHG-based sources is the enhancement of output intensity by breaking the limit of $10^{14} \mathrm{~W} / \mathrm{cm}^{2}[11-14]$ and thus becoming competitive with FEL-based sources. The temporal characterization of an IAP typically employs attosecond streaking $[15,16]$, i.e., measurement of the photoelectron spectrum produced by the IAP and a few-cycle mid-infrared pulse as a function of the time delay between the two pulses. Methods used to completely characterize the temporal intensity and phase of an IAP include frequency-resolved optical gating for complete reconstruction of attosecond bursts [17], phase retrieval by omega oscillation filtering (PROOF) [18], improved PROOF [19], "Volkov Transform" generalized projections algorithm (VTGPA) [20], multi-line VTGPA [8], phase retrieval of broadband pulses (PROBP) [21], and PROBP with autocorrelation [22]. All of these methods (except for iPROOF [19]) are based on the strong-field approximation for calculation of the photoelectron spectrum produced by the probe pulse at each step of the iterative reconstruction procedure. Although attosecond streaking methods are well established for reconstruction of IAP envelopes and even their carrier-envelope phases [23], photoelectron measurements are characterized by smaller detection efficiencies and lower signal-to-noise ratios than photon measurements. Hence, a

\footnotetext{
*Deceased.
}

few all-optical methods for characterization of an IAP have been proposed, including spatially-encoded arrangement for spectral phase interferometry for direct electric field reconstruction (SPIDER)- and extreme ultraviolet SPIDER [24], two in situ methods [25,26], and the recently developed double-blind holography method [27].

In this paper we propose an all-optical method for direct measurement of the temporal envelope of an IAP [produced by any source of an intense extreme ultraviolet (XUV) radiation] without the necessity for an iterative reconstruction procedure. It involves the HHG spectrum produced by an infrared (IR) laser pulse and a time-delayed XUV IAP. The method requires the detection of the harmonic signal in the energy region beyond the IR-driven plateau cutoff as a function of the time delay between the IR pulse and the IAP. Our analysis, which is based on an analytical parametrization of the HHG amplitude and numerical solution of the three-dimensional (3D) time-dependent Schrödinger equation (TDSE), shows that the harmonic yield as a function of time delay mimics the square of the IAP envelope, thereby providing a direct method for extracting the temporal envelope of the XUV IAP.

This paper is organized as follows. In Sec. II we discuss adiabatic results for the HHG amplitude in a strong IR field assisted by a weak IAP. We investigate factorization of HHG amplitude in the XUV-assisted channel in terms of laser factor and photorecombination amplitude and suggest a retrieval procedure for the IAP envelope from analysis of harmonic yield as a function of time delay between IR and XUV pulses. In Sec. III we analyze the accuracy of the suggested procedure by comparison of our analytical results with results obtained by numerical solution of the 3D TDSE for two configurations of the IR pulse: (1) a single-color IR pulse and (2) a twocolor IR pulse with orthogonally polarized components. Our results are summarized in Sec. IV. In Appendix A we present the explicit form of the laser factor for HHG amplitude in terms of ionization and recombination times. In Appendix B we provide mathematical justification for the uncertainty in 
recombination times. Atomic units (a.u.) are used throughout this paper unless specified otherwise.

\section{THEORETICAL BACKGROUND}

\section{A. Factorization of XUV-assisted HHG amplitude for the short IAP}

The XUV field can modify the IR-driven HHG process in two alternative ways. The first way consists in modification of the ionization step in the three-step scenario of HHG [28] and consists in replacing of tunneling ionization by the XUV single-photon ionization [29-32]. The second way is realized by absorbtion of the XUV photon at the moment of recombination [33]. In the latter case, the HHG spectrum produced by an intense IR pulse assisted by a weak (perturbative) XUV pulse includes additional plateaus extending beyond the usual HHG plateau produced by the intense IR pulse alone. These new plateaus stem from the additional channels made possible by the XUV pulse; e.g., absorption of an XUV photon at the moment of IR-field-driven electron recombination results in the formation of a two-plateau HHG spectrum with cutoff energies separated by the energy of the XUV photon [33].

As shown in Ref. [33], the XUV-assisted HHG amplitude for the case of a monochromatic XUV field can be factorized as the product of a laser-induced factor, $\tilde{a}_{1}\left(\Omega_{h}\right)$, describing tunneling and propagation in the intense IR field, and a two-photon (or Compton) recombination amplitude, $f_{\text {rec }}^{(1)}\left(E_{1}\right)$, corresponding to absorption of an XUV photon and emission of a harmonic photon of frequency $\Omega_{h}$ :

$$
\tilde{\mathcal{A}}_{1}\left(\Omega_{h}\right)=F_{\Omega} \tilde{a}_{1}\left(\Omega_{h}\right) f_{\text {rec }}^{(1)}\left(E_{1}\right),
$$

where $F_{\Omega}$ is the strength of the XUV field, $E_{1}=\Omega_{h}-I_{p}-$ $\Omega$ is the returning electron energy in the single-photon XUV channel, $I_{p}$ is the ionization potential of the atomic target, and $\Omega$ is the carrier frequency of the XUV field. ${ }^{1}$

The laser-induced factor $\tilde{a}_{1}\left(\Omega_{h}\right)$ describes the ionization and propagation steps of the three-step HHG scenario. For a monochromatic XUV field, it mimics the behavior of $a_{0}\left(\Omega_{h}\right)$ (i.e., the laser-induced factor for the IR field alone), $\tilde{a}_{1}\left(\Omega_{h}\right)=$ $a_{0}\left(\Omega_{h}-\Omega\right)$ [33]. In the low-frequency approximation within time-dependent effective range (TDER) theory, the factor $a_{0}\left(\Omega_{h}\right)$ can be presented as a twofold integral over the times $t^{\prime}$ and $t$ associated with ionization and recombination times in the three-step HHG scenario [33]:

$$
a_{0}\left(\Omega_{h}\right)=\frac{\mathcal{C}_{0}}{\sqrt{2 \pi i}} \frac{1}{2 \pi} \int_{-\infty}^{\infty} d t \int_{-\infty}^{t} d t^{\prime} \frac{e^{i \Omega_{h} t-i \mathcal{S}\left(t, t^{\prime}\right)}}{\left(t-t^{\prime}\right)^{3 / 2}},
$$

where $\mathcal{C}_{0}$ is the dimensionless asymptotic coefficient in the field-free wave function of a bound $s$ state at large distances, $\mathcal{S}\left(t, t^{\prime}\right)$ is the classical action

$$
\mathcal{S}\left(t, t^{\prime}\right)=I_{p}\left(t-t^{\prime}\right)+\frac{1}{2} \int_{t^{\prime}}^{t} \boldsymbol{P}^{2}\left(\xi ; t, t^{\prime}\right) d \xi,
$$

\footnotetext{
${ }^{1}$ It should be noted that although the analytical results for the XUV-assisted laser factor were reported only for the case of a linearly polarized monochromatic IR field the explicit form of the IR vector potential $\mathbf{A}_{\mathrm{IR}}(t)$ was not used explicitly in the derivations presented in Ref. [33]. Thus the results of Ref. [33] are valid also for a short IR pulse.
}

and $\boldsymbol{P}\left(\xi ; t, t^{\prime}\right)$ is the instantaneous (at the moment $\xi$ ) momentum of an electron moving along a closed trajectory in the time interval $\left(t^{\prime}, t\right)$ in the IR laser field with vector potential $\mathbf{A}_{\text {IR }}(\xi)$ :

$$
\boldsymbol{P}\left(\xi ; t, t^{\prime}\right)=\frac{1}{c}\left[\mathbf{A}_{\mathrm{IR}}(\xi)-\frac{1}{t-t^{\prime}} \int_{t^{\prime}}^{t} \mathbf{A}_{\mathrm{IR}}\left(\xi^{\prime}\right) d \xi^{\prime}\right] .
$$

In the quasiclassical approximation, the atomic factor $a_{0}\left(\Omega_{h}\right)$ can be presented in terms of real electron trajectories [34-36]:

$$
a_{0}\left(\Omega_{h}\right)=\sum_{j} Q_{j} a_{0}^{(j)}\left(\Omega_{h}\right)
$$

where $a_{0}^{(j)}\left(\Omega_{h}\right)$ is the TDER partial laser factor for the $j$ th closed classical electron trajectory in the IR field and $Q_{j}$ is the Coulomb factor, which extends the TDER results to the case of real atomic systems [37]. The explicit form of the factors $a_{0}^{(j)}$ and $Q_{j}$ can be found in Appendix A.

In order to generalize these results to the case of a short XUV pulse, we employ the following form for the electricfield vector of an XUV pulse:

$$
\begin{gathered}
\boldsymbol{F}_{\mathrm{XUV}}(t-\tau)=\hat{z} F_{\mathrm{XUV}} f_{\mathrm{XUV}}(t-\tau) \cos [\Omega(t-\tau)], \\
f_{\mathrm{XUV}}(t-\tau)=\int_{-\infty}^{\infty} \widehat{f_{\mathrm{XUV}}}\left(\Omega^{\prime}\right) e^{-i \Omega^{\prime}(t-\tau)} d \Omega^{\prime},
\end{gathered}
$$

where $F_{\mathrm{XUV}}, \Omega, \tau$, and $f_{\mathrm{XUV}}(t)$ are, respectively, the amplitude, carrier frequency, time delay, and temporal envelope of the XUV pulse. We assume the Fourier transform $\widehat{f_{\mathrm{XUV}}}\left(\Omega^{\prime}\right)$ has a pronounced maximum near $\Omega^{\prime}=0$. Then replacing $F_{\Omega} \rightarrow F_{\mathrm{XUV}} \widehat{f_{\mathrm{XUV}}}\left(\Omega^{\prime}\right) e^{i\left(\Omega^{\prime}+\Omega\right) \tau}$ in Eq. (1) HHG amplitude for the short XUV pulse can be found as a Fourier transform of the "monochromatic" amplitude $\tilde{\mathcal{A}}_{1}\left(\Omega_{h}\right)$ :

$$
\begin{aligned}
\mathcal{A}_{1}\left(\Omega_{h}\right)= & \int_{-\infty}^{\infty} F_{\mathrm{XUV}} \widehat{f_{\mathrm{XUV}}}\left(\Omega^{\prime}\right) e^{i\left(\Omega^{\prime}+\Omega\right) \tau} \\
& \times \tilde{a}_{1}\left(\Omega_{h}-\Omega^{\prime}\right) f_{\mathrm{rec}}^{(1)}\left(E_{1}-\Omega^{\prime}\right) d \Omega^{\prime} .
\end{aligned}
$$

If the frequency profile of the XUV pulse, $\widehat{f_{\mathrm{XUV}}}\left(\Omega^{\prime}\right)$, has a distinct maximum near $\Omega^{\prime}=0$ and the two-photon recombination amplitude is a smooth function of the absorbed photon energy, so that

$$
\left|f_{\mathrm{XUV}}(t-\tau) f_{\mathrm{rec}}^{(1)}\left(\Omega_{h}-I_{p}-\Omega\right)\right| \gg\left|\frac{d f_{\mathrm{XUV}}}{d t} \frac{\partial f_{\mathrm{rec}}^{(1)}}{\partial \Omega}\right|,
$$

one can evaluate the integral in Eq. (8) by replacing the $\Omega^{\prime}$ dependent recombination amplitude by its value at the carrier frequency of the XUV pulse, i.e., at $\Omega^{\prime}=0$. This approximation results in the factorization of the HHG amplitude for a short time-delayed XUV pulse in terms of a two-photon recombination amplitude and a laser-induced factor:

$$
\begin{aligned}
& \mathcal{A}_{1}\left(\Omega_{h}\right)=F_{\mathrm{XUV}} e^{i \Omega \tau} a_{1}\left(\Omega_{h}\right) f_{\mathrm{rec}}^{(1)}\left(E_{1}\right), \\
& a_{1}\left(\Omega_{h}\right)= \frac{\mathcal{C}_{0}}{\sqrt{2 \pi i}} \frac{1}{2 \pi} \int_{-\infty}^{\infty} d t \int_{-\infty}^{t} d t^{\prime} \frac{e^{i\left(\Omega_{h}-\Omega\right) t-i \mathcal{S}\left(t, t^{\prime}\right)}}{\left(t-t^{\prime}\right)^{3 / 2}} \\
& \times \int_{-\infty}^{\infty} \widehat{f_{\mathrm{XUV}}}\left(\Omega^{\prime}\right) e^{-i \Omega^{\prime}(t-\tau)} d \Omega^{\prime},
\end{aligned}
$$

where the last integral in (11) is the temporal profile (7) of the XUV pulse. Thus, the final form of the laser-induced factor 
for the short IR and XUV pulses within TDER theory is

$$
\begin{aligned}
a_{1}\left(\Omega_{h}\right)= & \frac{\mathcal{C}_{0}}{\sqrt{2 \pi i}} \frac{1}{2 \pi} \int_{-\infty}^{\infty} d t \int_{-\infty}^{t} d t^{\prime} \frac{e^{i\left(\Omega_{h}-\Omega\right) t-i \mathcal{S}\left(t, t^{\prime}\right)}}{\left(t-t^{\prime}\right)^{3 / 2}} \\
& \times f_{\mathrm{XUV}}(t-\tau)
\end{aligned}
$$

where $\mathcal{S}\left(t, t^{\prime}\right)$ is given by Eq. (3). Comparing Eqs. (2) and (12) and taking into account Eq. (5), the laser factor $a_{1}\left(\Omega_{h}\right)$ within the quasiclassical approximation may be presented in terms of $a_{0}^{(j)}\left(\Omega_{h}-\Omega\right)$ as follows:

$$
a_{1}\left(\Omega_{h}\right)=\sum_{j} Q_{j} a_{0}^{(j)}\left(\Omega_{h}-\Omega\right) f_{\mathrm{XUV}}\left(t_{r}^{(j)}-\tau\right),
$$

where $t_{r}^{(j)}$ is the recombination time for the $j$ th classical trajectory. The explicit form of the laser factor $a_{1}\left(\Omega_{h}\right)$ indicates that HHG yield on the XUV-induced plateau is maximized for time delay coinciding with the recombination time of the electron in the IR field, while in previous studies of XUV-assisted HHG [29-32] the time delay was tuned to the ionization time, which ensured maximal effects of IAPs on harmonics generated in the IR plateau.

Since our basic result (13) was obtained within quasiclassical approximation, we provide an estimate for the accuracy of this approximation, which assumes (1) smooth behavior of $f_{\text {rec }}^{(1)}\left(E_{1}\right)$ as a function of $\Omega$ [see discussion of Eq. (9)] and (2) the applicability of the classical trajectories approximation. Condition 2 gives a restriction on the temporal profile $f_{\mathrm{XUV}}(t)$; i.e., the characteristic time scale of the XUV envelope, $\left|f_{\mathrm{XUV}} /\left(d f_{\mathrm{XUV}} / d t\right)\right|$, at the recombination time $t_{r}^{(j)}-\tau$ should exceed the uncertainty in the recombination time $t_{r}^{(j)}$. This uncertainty can be estimated using the time-energy uncertainty principle, $\delta \mathcal{E} \delta t \sim 1$. If $\mathcal{E}(t)$ is the classical dependence of the electron energy (in the field of the IR pulse) on the recombination time [28], its variation is given by the first derivative, $\delta \mathcal{E}=\dot{\mathcal{E}}(t) \delta t \approx F_{\mathrm{IR}}^{2} \delta t /(4 \omega)$, for harmonics in the middle part of the HHG plateau, and by the second derivative, $\delta \mathcal{E}(t)=\ddot{\mathcal{E}}(t) \delta t^{2} / 2 \approx F_{\mathrm{IR}}^{2} \delta t^{2} / 6$, for cutoff harmonics [since $\dot{\mathcal{E}}(t)$ tends to zero there] [34,35]. Thus, according to the uncertainty principle, we find

$$
\begin{aligned}
& \delta t=\left[1 / \dot{\mathcal{E}}\left(t_{r}^{(j)}\right)\right]^{1 / 2} \approx \sqrt{4 \omega / F_{\mathrm{IR}}^{2}}, \quad \dot{\mathcal{E}}\left(t_{r}^{(j)}\right) \neq 0, \\
& \delta t=\left[1 / \ddot{\mathcal{E}}\left(t_{r}^{(j)}\right)\right]^{1 / 3} \approx\left(6 / F_{\mathrm{IR}}^{2}\right)^{1 / 3}, \quad \dot{\mathcal{E}}\left(t_{r}^{(j)}\right)=0,
\end{aligned}
$$

where $F_{\mathrm{IR}}$ and $\omega$ are the characteristic peak field strength and carrier frequency of the IR pulse. (See Appendix B for more details.)

\section{B. Retrieval procedures}

Using (13), the yield of harmonics on the XUV-induced plateau, $\mathcal{Y}_{1}\left(\Omega_{h}, \tau\right)$, is given by

$$
\begin{aligned}
\mathcal{Y}_{1}\left(\Omega_{h}, \tau\right)= & \left|\mathcal{A}_{1}\left(\Omega_{h}\right)\right|^{2} \propto \sum_{j}\left|a_{1}^{(j)}\right|^{2} f_{\mathrm{XUV}}^{2}\left(t_{r}^{(j)}-\tau\right) \\
& +\sum_{j, j^{\prime}, j \neq j^{\prime}} a_{1}^{(j)}\left[a_{1}^{\left(j^{\prime}\right)}\right]^{*} f_{\mathrm{XUV}}\left(t_{r}^{(j)}-\tau\right) f_{\mathrm{XUV}}\left(t_{r}^{\left(j^{\prime}\right)}-\tau\right),
\end{aligned}
$$

where $a_{1}^{(j)} \equiv Q_{j} a_{0}^{(j)}\left(\Omega_{h}-\Omega\right)$. The interference term [second line of Eq. (15)] can be omitted if (1) the duration of the IAP, $T_{\mathrm{XUV}}$, is much smaller than the minimum difference in recombination times, $T_{\mathrm{XUV}} \ll \min \left(\left|t_{r}^{(j)}-t_{r}^{\left(j^{\prime}\right)}\right|\right)$; or (2) the harmonic yield for a given energy $\Omega_{h}$ is due to a single closed classical trajectory. This suggests two alternative procedures for retrieval of the IAP pulse envelope by measuring the HHG yield vs time delay $\tau$.

Procedure 1. Choose a harmonic $\Omega_{h}$ associated with two or more well-separated electron trajectories. The harmonic yield as a function of time delay $\tau$ will then comprise a sequence of bursts at the recombination times $t_{r}^{(j)}$, separated by the time differences between recombination events, each of whose shapes replicates $f_{\mathrm{XUV}}^{2}\left(t_{r}^{(j)}-\tau\right)$. The relative height of each burst is given by $\left|a_{1}^{(j)}\right|^{2}$. To reconstruct the IAP temporal envelope, one should normalize all bursts to unity and then overlap the different scaled yields in order to form a single burst replicating the square of the XUV pulse envelope with higher accuracy than for a single $j$ th burst alone.

Procedure 2. Choose a harmonic $\Omega_{h}$ associated with a single electron trajectory with recombination time $t_{r}^{(0)}$. The harmonic yield vs time delay $\tau$ will then mimic the square of the XUV pulse envelope, $f_{\mathrm{XUV}}^{2}\left(t_{r}^{(0)}-\tau\right)$.

\section{NUMERICAL RESULTS}

We now proceed to illustrate retrieval procedures 1 and 2 by accurately calculating the XUV-assisted HHG spectrum for the hydrogen atom for two cases: a single-color linearly polarized IR pulse and a two-color IR pulse with linearly polarized orthogonal components.

\section{A. Single-color IR field}

For a single-color linearly polarized IR pulse, we represent the electric field of the pulse as

$$
\boldsymbol{F}_{\mathrm{IR}}(t)=\hat{z} F_{\mathrm{IR}} f_{\mathrm{IR}}(t) \cos \omega t,
$$

where its envelope is $f_{\mathrm{IR}}(t)=\sin ^{2}\left(\pi t / T_{\mathrm{IR}}\right)$ for $0<t<T_{\mathrm{IR}}$ and zero otherwise, and $T_{\mathrm{IR}}$ is its total duration. The XUV pulse is parametrized according to Eq. (6).

Numerical calculations were carried out for a fivecycle IR pulse $\left(T_{\mathrm{IR}}=20 \mathrm{fs}\right)$ with peak intensity $I_{\mathrm{IR}}=2 \times$ $10^{14} \mathrm{~W} / \mathrm{cm}^{2}, \omega=1 \mathrm{eV}$, and an XUV pulse with $\Omega=41 \mathrm{eV}$ and $I_{\mathrm{XUV}}=2 \times 10^{14} \mathrm{~W} / \mathrm{cm}^{2}$ for (1) a Gaussian envelope,

$$
\begin{aligned}
f_{\mathrm{XUV}}(t) & =f_{G}(t), \\
f_{G}(t) & =\exp \left[-\frac{2 \ln 2 t^{2}}{T_{\mathrm{XUV}}^{2}}\right],
\end{aligned}
$$

with XUV pulse durations (full width at half maximum of the intensity) of $T_{\mathrm{XUV}}=202$ and 303 as (two- and three-cycle pulses); and (2) a tailored pulse with the envelope given by two shifted Gaussian functions:

$$
f_{\mathrm{XUV}}(t)=f_{G}\left(t-\tau_{-}\right)+2 f_{G}\left(t-\tau_{+}\right) / 3,
$$

where $\tau_{ \pm}= \pm 4 \pi / \Omega$. For the single-color linearly polarized IR field, the 3D TDSE was solved for the Coulomb potential by expanding the wave function in spherical harmonics [38]. 


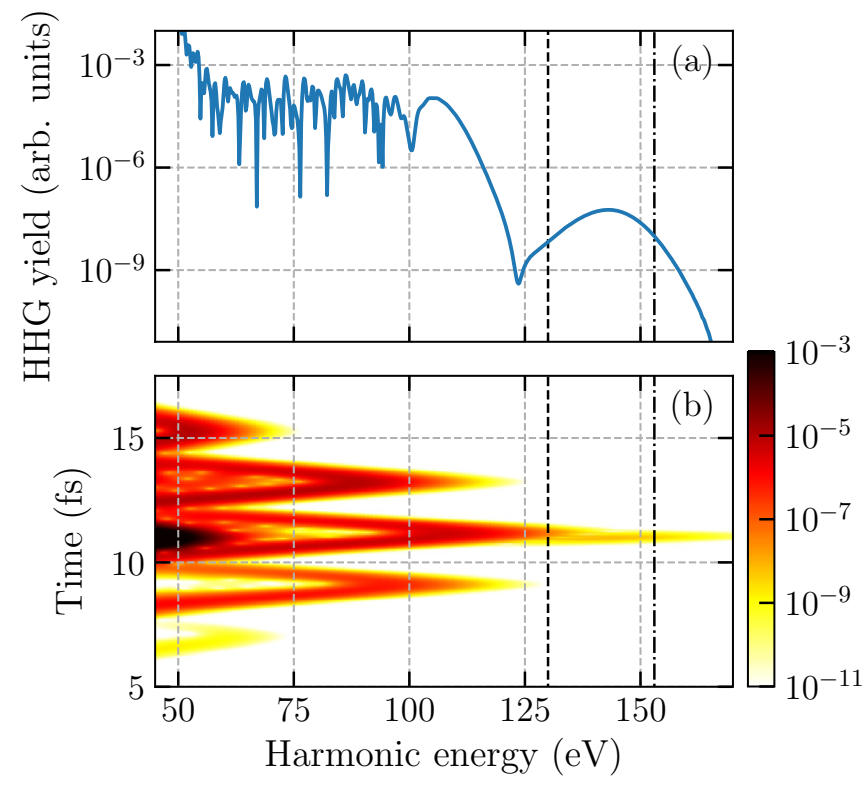

FIG. 1. (a) HHG yield for a five-cycle IR pulse field (16), with $T_{\mathrm{IR}}=20 \mathrm{fs}$ and $\omega=1 \mathrm{eV}$, and a Gaussian XUV pulse (17), with frequency $\Omega=41 \mathrm{eV}, T_{\mathrm{XUV}}=202$ as, and $\tau=10.95 \mathrm{fs}$. The IR and XUV pulse peak intensities are the same: $I_{\mathrm{IR}}=I_{\mathrm{XUV}}=$ $2 \times 10^{14} \mathrm{~W} / \mathrm{cm}^{2}$. Vertical dashed and dot-dashed lines indicate the harmonic energies $\Omega_{h}=130$ and $153 \mathrm{eV}$. (b) Color-coded timefrequency distribution of the HHG yield in (a).

In Fig. 1(a) we present the HHG spectrum for a Gaussian XUV pulse (17) with $T_{\mathrm{XUV}}=202$ as and a time delay $\tau \approx$ 10.95 fs. As shown in Fig. 1, the XUV field induces a second plateau. To retrieve the XUV pulse envelope, we choose two harmonic spectrum energies, $\Omega_{h}=130$ and $153 \mathrm{eV}$ (see Fig. 1). In principle, for $\Omega_{h}=130 \mathrm{eV}$ two (long and short) trajectories, the recombination times of which differ by $\approx 0.8 \mathrm{fs}$, may contribute. However, as is seen from Fig. 1(b), only the single trajectory contributes for $\Omega_{h}=130 \mathrm{eV}$ because the duration of the XUV pulse is much less than 0.8 fs. For $\Omega_{h}=153 \mathrm{eV}$ a single trajectory contributes, since its energy lies beyond the XUV-induced plateau cutoff.

In Fig. 2(a) we present the harmonic yield for $\Omega_{h}=130 \mathrm{eV}$ for a Gaussian XUV pulse envelope (17) as a function of time delay, $\tau-\tau_{0}$, where $\tau_{0} \approx 11.15$ fs is the return time for the extreme closed classical trajectory contributing to the formation of the cutoff harmonic. There are two bursts, separated by the difference in recombination times for short and long trajectories. The relative heights of the two bursts are given by the corresponding magnitudes $\left|a_{1}^{(j)}\right|^{2}$ for the long and short trajectories. To retrieve the XUV pulse envelope, we use Procedure 1 and scale both bursts to the same height [see the gray solid circles in Fig. 2(a)] and then overlap the burst on the right to the one on the left (see the parallel arrows pointing left). As a result, the set of transferred points (blue circles) and the points for the first pulse (red triangles) perfectly reproduce the original shape of the XUV pulse envelope. Note that the accuracy of this retrieval method decreases as the XUV pulse duration approaches the difference between recombination times of short and long trajectories (which increases with

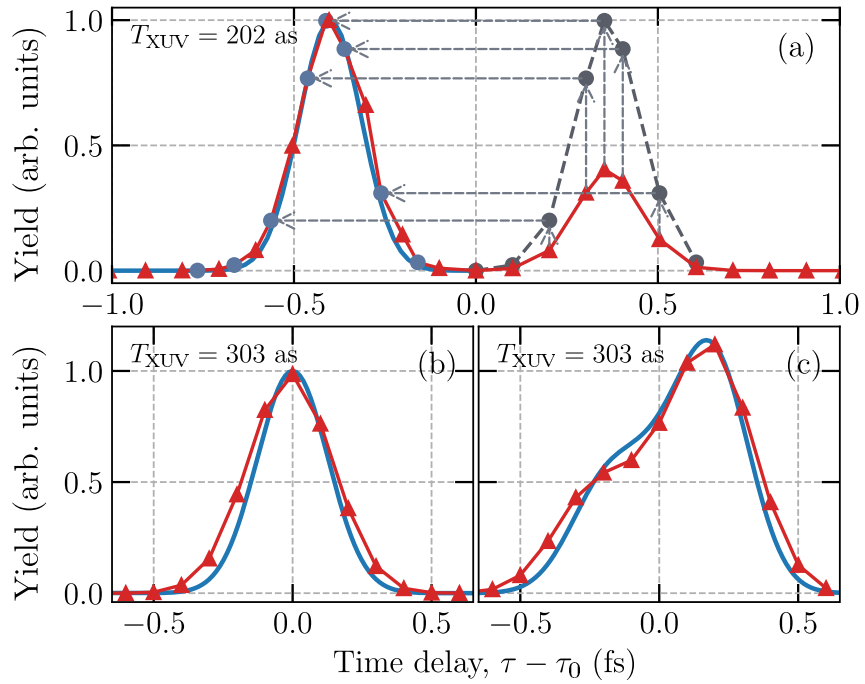

FIG. 2. An illustration of retrieval Procedure 1 (a) and Procedure 2 (b, c). Red lines with triangles show TDSE results for dependence of HHG yield for harmonic energy $\Omega_{h}=130 \mathrm{eV}$ (a) and $\Omega_{h}=$ $153 \mathrm{eV}\left(\mathrm{b}, \mathrm{c}\right.$ ) on the time delay $\tau-\tau_{0}$ (where $\tau_{0}=11.15$ as) between the single-color IR pulse (16) and Gaussian XUV pulse (17) with $T_{\mathrm{XUV}}=202$ as (a) and 303 as (b) and XUV pulse (18) with $T_{\mathrm{XUV}}=$ 303 as (c). Blue solid curves show original XUV pulse envelopes. The dashed gray curve with circles in (a) shows scaled dependence of HHG yield on the time delay; blue solid circles show shifted data points (see text for details). Carrier frequencies and intensities of the IR and XUV pulses are the same as in Fig. 1.

increasing wavelength of the IR pulse); it also has a lower limit for the XUV pulse duration given by the "resolution" time, $\delta t$ [see Eq. (14a)].

In Figs. 2(b) and 2(c) we present the dependence of the harmonic yield on the time delay $\tau-\tau_{0}$ for the harmonic $\Omega_{h}=153 \mathrm{eV}$ and XUV pulses (17) and (18), respectively, with $T_{\mathrm{XUV}}=303$ as. For XUV pulses with $T_{\mathrm{XUV}}=303$ as, the harmonic yield as a function of time delay accurately reproduces the square of the XUV pulse envelope, $f_{\mathrm{XUV}}^{2}(t)$, for both symmetric and asymmetric XUV IAPs. For shorter IAPs (e.g., $T_{\mathrm{XUV}}=202$ as), however, the retrieved envelope is much broader than that of the XUV pulse owing to the comparable magnitudes of the XUV pulse duration and the resolution time, $\delta t \approx 246$ as, for the cutoff harmonic [see Eq. (14b)]. Thus, for a single-color IR pulse, the single trajectory Procedure 2 based on the cutoff harmonic has much smaller accuracy than Procedure 1 based on the plateau harmonic.

\section{B. Two-color IR field with orthogonal polarizations}

Procedure 2 can work, however, by using an alternative IR field waveform that supports only a single trajectory contribution to the HHG spectrum. Such a waveform is realized for a two-color field with linearly polarized, mutually perpendicular components $[39,40]$ :

$$
\boldsymbol{F}_{\mathrm{IR}}(t)=F_{\mathrm{IR}} f_{\mathrm{IR}}(t)[\hat{z} \cos \omega t-\beta \hat{\boldsymbol{x}} \sin 2 \omega t],
$$

where $\beta=0.8$ is the field strength ratio for the second and first harmonics. In this case any harmonics in the middle part 


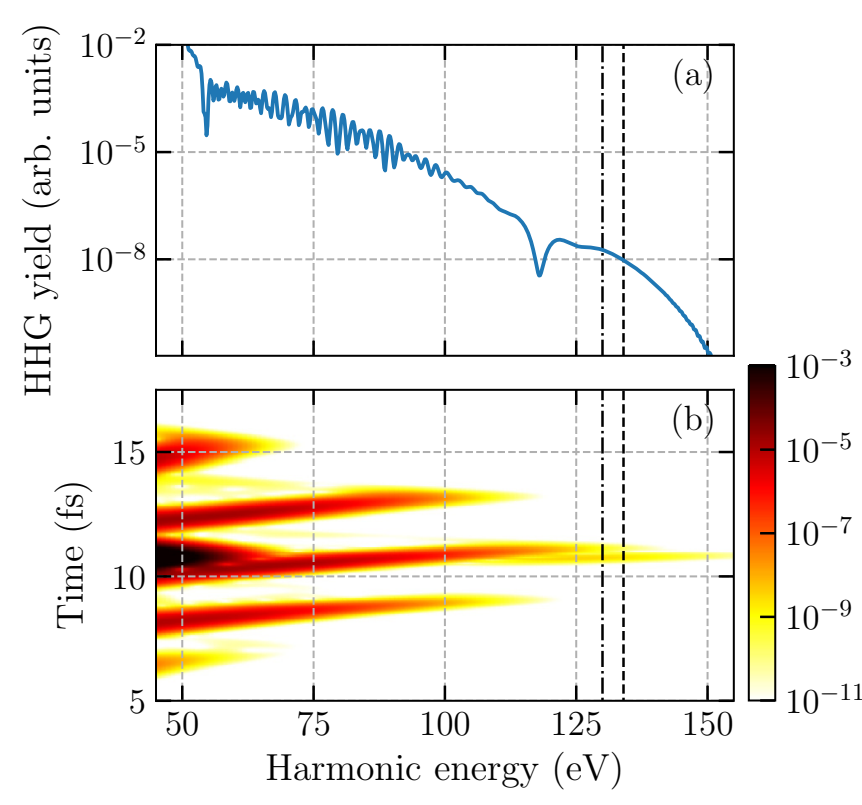

FIG. 3. (a) HHG spectrum for a two-color IR field (19) with $\beta=0.8$ and a two-cycle XUV pulse (17) $\left(T_{\mathrm{XUV}}=202\right.$ as). Carrier frequencies and intensities are the same as in Fig. 1. Vertical dashed lines indicate the energy positions $\Omega_{h}=130$ and $134 \mathrm{eV}$. (b) Colorcoded time-frequency distribution of the HHG yield for the same parameters as in (a).

of the plateau can be used to map the XUV pulse envelope. The characteristic resolution time for harmonics in the middle part of the plateau is determined by both the intensity and the frequency of the IR field, $\delta t \approx \sqrt{4 \omega / F_{\mathrm{IR}}^{2}}$. Since the frequency of the IR field is small, the resolution of the retrieval Procedure 2 for the two-color IR field (19) increases in comparison with that for the single-color case (16).

To demonstrate the Procedure 2 retrieval accuracy for the two-color IR pulse (19), we calculate the XUV-assisted HHG spectrum for a carrier frequency $\omega=1 \mathrm{eV}$ and peak intensity $I_{\mathrm{IR}}=2 \times 10^{14} \mathrm{~W} / \mathrm{cm}^{2}$. Unlike the case of a single-color linearly polarized IR pulse, we solve the 3D TDSE in Cartesian coordinates by a split-step method with a fast Fourier transform $[40,41]$. To speed up the computations, rather large spatial steps were used (0.325 a.u.). Therefore, in order to obtain the correct $\mathrm{H}$ atom binding energy, $I_{p}=13.65 \mathrm{eV}$, we employed a soft-Coulomb potential, $U(r)=-\alpha \operatorname{sech}^{2}(r / a)-$ $\tanh (r / a) / r$ with $\alpha=0.3$ and $a=2.17$ [40,41]. The characteristic resolution time interval (14a) for the above IR pulse parameters is $\delta t \approx 123$ as. The HHG spectrum for the abovespecified IR pulse and a two-cycle Gaussian XUV pulse (17) with time delay $\tau_{0}=10.73$ fs (corresponding to the recombination time for the harmonic with energy $130 \mathrm{eV}$ ) is shown in Fig. 3(a). The harmonics involving the absorption of the XUV photon lie in the energy range $120<\Omega_{h}<$ $150 \mathrm{eV}$ [see Fig. 3(b)]. We choose two harmonics from the middle of this interval (see Fig. 3) to retrieve the XUV pulse envelope.

In Fig. 4 we present the dependence of the harmonic yield on the time delay $\tau-\tau_{0}$ for two harmonics from the plateau region in Fig. 3(a), $\Omega_{h}=130$ and $134 \mathrm{eV} ; \tau_{0}=10.73 \mathrm{fs}$ is the return time for the shortest electron trajectory for the harmonic

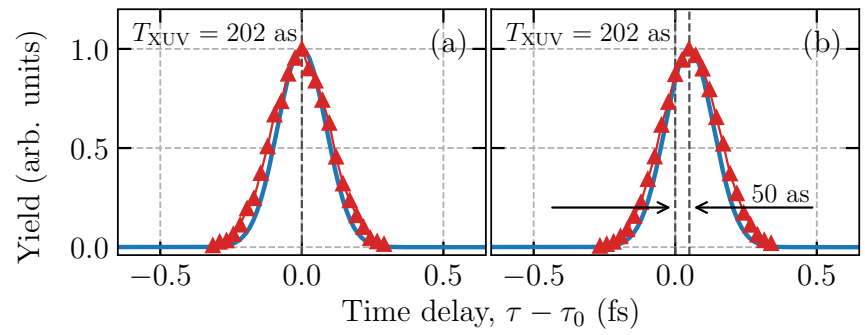

FIG. 4. Dependence of HHG yields on time delay $\tau-\tau_{0}$ (where $\tau_{0}=10.73 \mathrm{fs}$ ) for harmonic energies (a) $\Omega_{h}=130 \mathrm{eV}$ and (b) $\Omega_{h}=$ $134 \mathrm{eV}$ in the XUV-assisted HHG spectrum in Fig. 3. Intensities and carrier frequencies are the same as in Fig. 1. The XUV pulse duration is $T_{\mathrm{XUV}}=202$ as. Red lines with triangles show the retrieved square of the XUV pulse envelope; solid blue lines show the square of the original XUV pulse envelope.

with $\Omega_{h}=130 \mathrm{eV}$. One observes good agreement between the retrieved and original XUV pulse envelopes. Note that the position of the maximum for the retrieved pulse for $\Omega_{h}=$ $134 \mathrm{eV}$ in Fig. 4(b) is shifted by 50 as with respect to that for $\Omega_{h}=130 \mathrm{eV}$ in Fig. 4(a). This shift stems from the difference in recombination times for these two harmonics. Thus, the retrieval Procedure 2 using a two-color IR pulse (19) allows one to measure the difference in recombination times for the harmonics on an attosecond time scale, thereby providing an alternative procedure for recollision time measurements (see Ref. [42]). Note also that one can overlap the scaled harmonic bursts for different harmonics similarly to Procedure 1 of overlapping different trajectory bursts for the same harmonic [as shown in Fig. 2(a)].

\section{CONCLUSION}

To conclude, we have proposed an all-optical method for reconstruction of an XUV IAP envelope from analysis of XUV-assisted harmonic yields beyond the IR-induced HHG plateau as a function of time delay between IR and XUV pulses. The resolution of the method depends on the position of the harmonic in the HHG spectrum: for harmonics on the slope of an XUV-induced plateau, the resolution is determined only by the intensity of the IR field, while for harmonics in the middle part of the plateau the resolution increases with decrease of the IR pulse carrier frequency. In addition, the proposed method makes possible a direct mapping of electron recombination time difference and relative contribution of closed classical trajectories to the HHG yield as functions of the harmonic energy. Finally, we note that experimental realization of proposed retrieval schemes requires accounting of medium effects [43-45], which can be minimized, so that the HHG yield can be reduced to a single atom response $[46,47]$. This makes possible realization of in situ methods $[25,26]$, measurement of ionization and recombination times [42], and realization of proposed retrieval procedures due to uniformity of nonlinear dynamics and propagation effects for a harmonic with frequency $\Omega_{h}-\Omega$ in the IR-plateau region and XUV-induced harmonic $\Omega_{h}$, which is given by the same factors $a_{0}^{(j)}$ [see Eqs. (5) and (13)]. 


\section{ACKNOWLEDGMENTS}

This work was supported in part by the Ministry of Education and Science of the Russian Federation through Grant No. 3.1659.2017/4.6, the Russian Science Foundation through Grant No. 18-12-00476 (numerical calculations), and by the U.S. National Science Foundation through Grant No. PHY-1505492 (A.F.S.). T.S.S. acknowledges support from the Foundation for the Advancement of Theoretical Physics and Mathematics "Basis" (Project No. 19-1-3-72-1).

\section{APPENDIX A: EXPLICIT EXPRESSIONS FOR THE ADIABATIC APPROXIMATION RESULT FOR THE LASER-INDUCED FACTOR}

The integrals in Eqs. (2) and (12) can be evaluated using the adiabatic (or low-frequency) approximation of Ref. [36]. In this approximation, the analytical result for the factors $a_{0}\left(\Omega_{h}\right)$ and $a_{1}\left(\Omega_{h}\right)$ can be presented in terms of real classical trajectories, Eqs. (5) and (13), defined by the corresponding real initial (ionization), $t_{i}^{(j)}$, and return (recombination), $t_{r}^{(j)}$, times [36]. These times are solutions of a system of transcendental equations:

$$
\begin{aligned}
\boldsymbol{K}_{j}^{\prime} \cdot \dot{\boldsymbol{K}}_{j}^{\prime} & =0, \\
\frac{\boldsymbol{K}_{j}^{2}}{2} & =E_{1}-\Delta \mathcal{E}_{j},
\end{aligned}
$$

where

$$
\Delta \mathcal{E}_{j}=-\frac{\boldsymbol{K}_{j}^{\prime 2}+\kappa^{2}}{2 \Delta t_{j}}\left[\frac{2 \frac{\boldsymbol{K}_{j} \cdot \boldsymbol{K}_{j}^{\prime}}{\Delta t_{j}}-\boldsymbol{F}_{j}^{\prime} \cdot\left(\boldsymbol{K}_{j}-\boldsymbol{K}_{j}^{\prime}\right)}{\boldsymbol{F}_{j}^{\prime 2}-\boldsymbol{K}_{j}^{\prime} \cdot \dot{\boldsymbol{F}}_{j}^{\prime}}\right],
$$

and $E_{1}$ is the returning electron energy $\left[E_{1}=\Omega_{h}-I_{p}\right.$ for $a_{0}\left(\Omega_{h}\right)$ and $E_{1}=\Omega_{h}-I_{p}-\Omega$ for $\left.a_{1}\left(\Omega_{h}\right)\right]$, $\Delta t_{j}=t_{r}^{(j)}-t_{i}^{(j)}, \quad \kappa=\sqrt{2 I_{p}}, \quad \boldsymbol{K}_{j}^{\prime} \equiv \boldsymbol{P}\left(t_{i}^{(j)} ; t_{r}^{(j)}, t_{i}^{(j)}\right)$, $\dot{\boldsymbol{K}}_{j}^{\prime} \equiv \partial \boldsymbol{P}\left(t_{i}^{(j)} ; t_{r}^{(j)}, t_{i}^{(j)}\right) / \partial t_{i}^{(j)}, \quad \boldsymbol{K}_{j} \equiv \boldsymbol{P}\left(t_{r}^{(j)} ; t_{r}^{(j)}, t_{i}^{(j)}\right), \quad \boldsymbol{F}_{j}^{\prime} \equiv$ $\boldsymbol{F}_{\mathrm{IR}}\left(t_{i}^{(j)}\right), \quad \dot{\boldsymbol{F}}_{j}^{\prime} \equiv \dot{\boldsymbol{F}}_{\mathrm{IR}}\left(t_{i}^{(j)}\right), \quad$ and $\quad \boldsymbol{F}_{\mathrm{IR}}(t)=-\partial \boldsymbol{A}_{\mathrm{IR}}(t) / \partial(c t)$. As shown in Ref. [36], $a_{0}^{(j)}\left(\Omega_{h}\right)$ [and corresponding $\left.a_{1}^{(j)}\left(\Omega_{h}\right)=a_{0}^{(j)}\left(\Omega_{h}-\Omega\right)\right]$ has an explicit form in terms of real classical times $t_{r}^{(j)}$ and $t_{i}^{(j)}$ :

$$
a_{0}^{(j)}\left(\Omega_{h}\right)=a_{j}^{(\text {tun })} a_{j}^{(\text {prop })}\left(\Omega_{h}\right),
$$

where the propagation factor, $a_{j}^{(\mathrm{prop})}$, is given by

$$
a_{j}^{(\mathrm{prop})}\left(\Omega_{h}\right)=i \frac{e^{-i \mathcal{S}\left(t_{r}^{(j)}, t_{i}^{(j)}\right)+i \Omega_{h} t_{r}^{(j)}}}{\Delta t_{j}^{3 / 2} \sqrt{\boldsymbol{K}_{j} \cdot \dot{\boldsymbol{K}}_{j}}}
$$

where $\dot{\boldsymbol{K}}_{j} \equiv \partial \boldsymbol{P}\left(t_{r}^{(j)} ; t_{r}^{(j)}, t_{i}^{(j)}\right) / \partial t_{r}^{(j)}$ and where the tunneling factor, $a_{j}^{(\text {tun })}$, is given by

$$
a_{j}^{\text {(tun) }}=\frac{\mathcal{C}_{0}}{\pi} \sqrt{\frac{\kappa}{2}} \frac{e^{-\frac{\varkappa_{j}^{3}}{3 \mathcal{F}_{j}}}}{\sqrt{\varkappa_{j} \mathcal{F}_{j}}},
$$

where

$$
\mathcal{F}_{j}=\sqrt{\boldsymbol{F}_{j}^{\prime 2}-\boldsymbol{K}_{j}^{\prime} \cdot \dot{\boldsymbol{F}}_{j}^{\prime}}, \quad \varkappa_{j}=\sqrt{2 I_{p}+\boldsymbol{K}_{j}^{\prime 2}} .
$$

The Coulomb factor, $Q_{j}$, in Eqs. (5) and (13) consists of two factors [37]:

$$
\begin{aligned}
Q_{j} & =Q_{\text {stat }}^{(j)} R^{(j)}, \\
Q_{\text {stat }}^{(j)} & =\left(\frac{2 \kappa^{3}}{F_{j}^{\prime}}\right)^{Z / \kappa}, \quad F_{j}^{\prime}=\sqrt{\boldsymbol{F}_{j}^{\prime 2}}, \\
R_{j} & =\left[\frac{2 F_{j}^{\prime}}{\mathcal{F}_{j}\left(\sqrt{1+\frac{\boldsymbol{K}_{j}^{\prime 2}}{\kappa^{2}}}+\frac{2}{\sqrt{3}} \sqrt{1-\frac{\boldsymbol{F}_{j}^{\prime 2}}{4 \mathcal{F}_{j}^{2}}}\right)}\right]^{Z / \kappa},
\end{aligned}
$$

where $Z$ is the charge of the residual atomic core.

\section{APPENDIX B: DERIVATION OF QUANTUM UNCERTAINTIES [EQS. (14)]}

The adiabatic approach is justified by the smoothness of the preexponential factors compared to the rapidly oscillating exponential function in Eq. (12). In particular, the characteristic time scale of the XUV envelope, $\left|f_{\mathrm{XUV}} /\left(d f_{\mathrm{XUV}} / d t\right)\right|$, at the time $t_{r}^{(j)}-\tau$ should exceed the vicinity of the recombination time $t_{r}^{(j)}$, which gives the main contribution to the integral in Eq. (12). This vicinity, $\delta t$, is determined by the second derivative of the action $\mathcal{S}\left(t, t^{\prime}\right)$ at the recombination time $t_{r}^{(j)}$ :

$$
\delta t=\left[\frac{1}{2} \frac{\partial^{2} S\left(t_{r}^{(j)}, t_{i}^{(j)}\right)}{\left(\partial t_{r}^{(j)}\right)^{2}}\right]^{-1 / 2}
$$

where

$$
\frac{\partial^{2} S\left(t_{r}^{(j)}, t_{i}^{(j)}\right)}{\left(\partial t_{r}^{(j)}\right)^{2}}=\boldsymbol{K}_{j} \cdot \dot{\boldsymbol{K}}_{j}=\alpha_{j} F_{\mathrm{IR}}^{2} / \omega .
$$

The parameter $\alpha_{j}$ differs only slightly from one trajectory to another; its value lies in the interval $0.45-0.55$ for both a linearly polarized IR pulse and a two-color IR pulse with linearly polarized mutually perpendicular components. Setting $\alpha_{j} \approx 0.5$, Eqs. (B1) and (B2) give Eq. (14a) of the main text for $\delta t$, which may be interpreted as a quantum uncertainty in the recombination time.

Near the high-energy cutoff, the second derivative of the action tends to zero, so that the integration in (12) must be treated in an alternate way [35]. In this case, the vicinity of the extreme recombination time is given by the third derivative of the action:

$$
\delta t=\left[\frac{1}{6} \frac{\partial^{3} S\left(t_{r}^{(j)}, t_{i}^{(j)}\right)}{\left(\partial t_{r}^{(j)}\right)^{3}}\right]^{-1 / 3}
$$

where

$$
\frac{\partial^{3} S\left(t_{r}^{(j)}, t_{i}^{(j)}\right)}{\left(\partial t_{r}^{(j)}\right)^{3}}=\boldsymbol{K}_{j} \cdot \ddot{\boldsymbol{K}}_{j}+\dot{\boldsymbol{K}}_{j}{ }^{2}=\delta_{j} F_{\mathrm{IR}}^{2},
$$

where $\ddot{\boldsymbol{K}}_{j} \equiv \partial^{2} \boldsymbol{P}\left(t_{r}^{(j)} ; t_{r}^{(j)}, t_{i}^{(j)}\right) /\left(\partial t_{r}^{(j)}\right)^{2}$. In Eq. (B4), the spreading factor, $\delta_{j}$, is $\approx 1$ for both a linearly polarized IR pulse and a two-color IR pulse with linearly polarized mutually perpendicular components. Setting $\delta_{j} \approx 1$, Eqs. (B3) and (B4) give Eq. (14b) of the main text. 
[1] M. Hentschel, R. Kienberger, C. Spielmann, G. A. Reider, N. Milosevic, T. Brabec, P. Corkum, U. Heinzmann, M. Drescher, and F. Krausz, Attosecond metrology, Nature (London) 414, 509 (2001).

[2] A. L. Cavalieri, N. Müller, T. Uphues, V. S. Yakovlev, A. Baltuška, B. Horvath, B. Schmidt, L. Blümel, R. Holzwarth, S. Hendel, M. Drescher, U. Kleineberg, P. M. Echenique, R. Kienberger, F. Krausz, and U. Heinzmann, Attosecond spectroscopy in condensed matter, Nature (London) 449, 1029 (2007).

[3] G. Sansone, F. Kelkensberg, J. F. Pérez-Torres, F. Morales, M. F. Kling, W. Siu, O. Ghafur, P. Johnsson, M. Swoboda, E. Benedetti, F. Ferrari, F. Lépine, J. L. Sanz-Vicario, S. Zherebtsov, I. Znakovskaya, A. L'Huillier, M. Y. Ivanov, M. Nisoli, F. Martín, and M. J. J. Vrakking, Electron localization following attosecond molecular photoionization, Nature (London) 465, 763 (2010).

[4] M. Schultze, M. Fieß, N. Karpowicz, J. Gagnon, M. Korbman, M. Hofstetter, S. Neppl, A. L. Cavalieri, Y. Komninos, T. Mercouris, C. A. Nicolaides, R. Pazourek, S. Nagele, J. Feist, J. Burgdörfer, A. M. Azzeer, R. Ernstorfer, R. Kienberger, U. Kleineberg, E. Goulielmakis, F. Krausz, and V. S. Yakovlev, Delay in photoemission, Science 328, 1658 (2010).

[5] E. Goulielmakis, Z.-H. Loh, A. Wirth, R. Santra, N. Rohringer, V. S. Yakovlev, S. Zherebtsov, T. Pfeifer, A. M. Azzeer, M. F. Kling, S. R. Leone, and F. Krausz, Real-time observation of valence electron motion, Nature (London) 466, 739 (2010).

[6] F. Calegari, D. Ayuso, A. Trabattoni, L. Belshaw, S. De Camillis, S. Anumula, F. Frassetto, L. Poletto, A. Palacios, P. Decleva, J. B. Greenwood, F. Martín, and M. Nisoli, Ultrafast electron dynamics in phenylalanine initiated by attosecond pulses, Science 346, 336 (2014).

[7] J. Li, X. Ren, Y. Yin, K. Zhao, A. Chew, Y. Cheng, E. Cunningham, Y. Wang, S. Hu, Y. Wu, M. Chini, and Z. Chang, 53-attosecond $\mathrm{X}$-ray pulses reach the carbon K-edge, Nat. Commun. 8, 186 (2017).

[8] T. Gaumnitz, A. Jain, Y. Pertot, M. Huppert, I. Jordan, F. Ardana-Lamas, and H. J. Wörner, Streaking of 43-attosecond soft-x-ray pulses generated by a passively CEP-stable midinfrared driver, Opt. Express 25, 27506 (2017).

[9] Z. Tibai, G. Tóth, M. I. Mechler, J. A. Fülöp, G. Almási, and J. Hebling, Proposal for Carrier-Envelope-Phase Stable SingleCycle Attosecond Pulse Generation in the Extreme-Ultraviolet Range, Phys. Rev. Lett. 113, 104801 (2014).

[10] A. Mak, G. Shamuilov, P. Salén, D. Dunning, J. Hebling, Yu. Kida, R. Kinjo, B. W. J. McNeil, T. Tanaka, N. Thompson, Z. Tibai, G. Tóth, and V. Goryashko, Attosecond single-cycle undulator light: A review, Rep. Prog. Phys. 82, 025901 (2019).

[11] P. Tzallas, E. Skantzakis, L. A. A. Nikolopoulos, G. D. Tsakiris, and D. Charalambidis, Extreme-ultraviolet pump-probe studies of one-femtosecond-scale electron dynamics, Nat. Phys. 7, 781 (2011).

[12] E. J. Takahashi, P. Lan, O. D. Mücke, Y. Nabekawa, and K. Midorikawa, Attosecond nonlinear optics using gigawatt-scale isolated attosecond pulses, Nat. Commun. 4, 2691 (2013).

[13] P. A. Carpeggiani, P. Tzallas, A. Palacios, D. Gray, F. Martín, and D. Charalambidis, Disclosing intrinsic molecular dynamics on the 1-fs scale through extreme-ultraviolet pump-probe measurements, Phys. Rev. A 89, 023420 (2014).
[14] B. Senfftleben, M. Kretschmar, A. Hoffmann, M. Sauppe, J. Tümmler, I. Will, T. Nagy, M. J. J. Vrakking, D. Rupp, and B. Schütte, Highly nonlinear ionization of atoms induced by intense high-harmonic pulses, arXiv:1911.01375 (2019).

[15] J. Itatani, F. Quéré, G. L. Yudin, M. Yu. Ivanov, F. Krausz, and P. B. Corkum, Attosecond Streak Camera, Phys. Rev. Lett. 88, 173903 (2002).

[16] M. Kitzler, N. Milosevic, A. Scrinzi, F. Krausz, and T. Brabec, Quantum Theory of Attosecond XUV Pulse Measurement by Laser Dressed Photoionization, Phys. Rev. Lett. 88, 173904 (2002).

[17] Y. Mairesse and F. Quéré, Frequency-resolved optical gating for complete reconstruction of attosecond bursts, Phys. Rev. A 71, 011401(R) (2005).

[18] M. Chini, S. Gilbertson, S. D. Khan, and Z. Chang, Characterizing ultrabroadband attosecond lasers, Opt. Express 18, 13006 (2010).

[19] G. Laurent, W. Cao, I. Ben-Itzhak, and C. L. Cocke, Attosecond pulse characterization, Opt. Express 21, 16914 (2013).

[20] P. D. Keathley, S. Bhardwaj, J. Moses, G. Laurent, and F. X. Kärtner, Volkov transform generalized projection algorithm for attosecond pulse characterization, New J. Phys. 18, 073009 (2016)

[21] X. Zhao, H. Wei, Y. Wu, and C. D. Lin, Phase-retrieval algorithm for the characterization of broadband single attosecond pulses, Phys. Rev. A 95, 043407 (2017).

[22] W.-W. Yu, X. Zhao, H. Wei, S.-J. Wang, and C. D. Lin, Method for spectral phase retrieval of single attosecond pulses utilizing the autocorrelation of photoelectron streaking spectra, Phys. Rev. A 99, 033403 (2019).

[23] C. Liu, M. Reduzzi, A. Trabattoni, A. Sunilkumar, A. Dubrouil, F. Calegari, M. Nisoli, and G. Sansone, Carrier-Envelope Phase Effects of a Single Attosecond Pulse in Two-Color Photoionization, Phys. Rev. Lett. 111, 123901 (2013).

[24] E. Cormier, I. A. Walmsley, E. M. Kosik, A. S. Wyatt, L. Corner, and L. F. DiMauro, Self-Referencing, Spectrally, or Spatially Encoded Spectral Interferometry for the Complete Characterization of Attosecond Electromagnetic Pulses, Phys. Rev. Lett. 94, 033905 (2005).

[25] N. Dudovich, O. Smirnova, J. Levesque, Y. Mairesse, M. Yu. Ivanov, D. M. Villeneuve, and P. B. Corkum, Measuring and controlling the birth of attosecond XUV pulses, Nat. Phys. 2, 781 (2006).

[26] K. T. Kim, C. Zhang, A. D. Shiner, S. E. Kirkwood, E. Frumker, G. Gariepy, A. Naumov, D. M. Villeneuve, and P. B. Corkum, Manipulation of quantum paths for space-time characterization of attosecond pulses, Nat. Phys. 9, 159 (2013).

[27] O. Pedatzur, A. Trabattoni, B. Leshem, H. Shalmoni, M. C. Castrovilli, M. Galli, M. Lucchini, E. Månsson, F. Frassetto, L. Poletto, B. Nadler, O. Raz, M. Nisoli, F. Calegari, D. Oron, and N. Dudovich, Double-blind holography of attosecond pulses, Nat. Photon. 13, 91 (2019).

[28] P. B. Corkum, Plasma Perspective on Strong-Field Multiphoton Ionization, Phys. Rev. Lett. 71, 1994 (1993).

[29] K. J. Schafer, M. B. Gaarde, A. Heinrich, J. Biegert, and U. Keller, Strong Field Quantum Path Control using Attosecond Pulse Trains, Phys. Rev. Lett. 92, 023003 (2004).

[30] J. Biegert, A. Heinrich, C. P. Hauri, W. Kornelis, P. Schlup, M. P. Anscombe, M. B. Gaarde, K. J. Schafer, and U. Keller, Control 
of high-order harmonic emission using attosecond pulse trains, J. Mod. Opt. 53, 87 (2006).

[31] G. Gademann, F. Kelkensberg, W. K. Siu, P. Johnsson, M. B. Gaarde, K J Schafer, and M. J. J. Vrakking, Attosecond control of electron-ion recollision in high harmonic generation, New J. Phys. 13, 033002 (2011).

[32] D. Azoury, M. Krüger, G. Orenstein, H. R. Larsson, S. Bauch, B. D. Bruner, and N. Dudovich, Self-probing spectroscopy of XUV photo-ionization dynamics in atoms subjected to a strongfield environment, Nat. Commun. 8, 1453 (2017).

[33] T. S. Sarantseva, M. V. Frolov, N. L. Manakov, A. A. Silaev, N. V. Vvedenskii, and A. F. Starace, XUV-assisted high-orderharmonic-generation spectroscopy, Phys. Rev. A 98, 063433 (2018).

[34] M. V. Frolov, N. L. Manakov, T. S. Sarantseva, and A. F. Starace, Analytic formulae for high harmonic generation, J. Phys. B 42, 035601 (2009).

[35] M. V. Frolov, N. L. Manakov, A. M. Popov, O. V. Tikhonova, E. A. Volkova, A. A. Silaev, N. V. Vvedenskii, and A. F. Starace, Analytic theory of high-order-harmonic generation by an intense few-cycle laser pulse, Phys. Rev. A 85, 033416 (2012).

[36] M. V. Frolov, N. L. Manakov, A. A. Minina, A. A. Silaev, N. V. Vvedenskii, M. Yu. Ivanov, and A. F. Starace, Analytic description of high-order harmonic generation in the adiabatic limit with application to an initial $s$ state in an intense bicircular laser pulse, Phys. Rev. A 99, 053403 (2019).

[37] M. V. Frolov, N. L. Manakov, A. A. Minina, S. V. Popruzhenko, and A. F. Starace, Adiabatic-limit Coulomb factors for photoelectron and high-order-harmonic spectra, Phys. Rev. A 96, 023406 (2017).

[38] A. A. Silaev, A. A. Romanov, and N. V. Vvedenskii, Multihump potentials for efficient wave absorption in the numerical solution of the time-dependent Schrödinger equation, J. Phys. B 51, 065005 (2018).
[39] L. Brugnera, D. J. Hoffmann, T. Siegel, F. Frank, A. Zaïr, J. W. G. Tisch, and J. P. Marangos, Trajectory Selection in High Harmonic Generation by Controlling the Phase between Orthogonal Two-Color Fields, Phys. Rev. Lett. 107, 153902 (2011).

[40] M. V. Frolov, N. L. Manakov, T. S. Sarantseva, A. A. Silaev, N. V. Vvedenskii, and A. F. Starace, Control of threshold enhancements in harmonic generation by atoms in a two-color laser field with orthogonal polarizations, Phys. Rev. A 93, 023430 (2016).

[41] M. V. Frolov, N. L. Manakov, A. A. Minina, N. V. Vvedenskii, A. A. Silaev, M. Yu. Ivanov, and A. F. Starace, Control of Harmonic Generation by the Time Delay Between Two-Color, Bicircular Few-Cycle Mid-IR Laser Pulses, Phys. Rev. Lett. 120, 263203 (2018).

[42] D. Shafir, H. Soifer, B. D. Bruner, M. Dagan, Y. Mairesse, S. Patchkovskii, M. Yu. Ivanov, O. Smirnova, and N. Dudovich, Resolving the time when an electron exits a tunnelling barrier, Nature (London) 485, 343 (2012).

[43] P. Salières, A. L'Huillier, and M. Lewenstein, Coherence Control of High-Order Harmonics, Phys. Rev. Lett. 74, 3776 (1995).

[44] P. Balcou, P. Salières, A. L'Huillier, and M. Lewenstein, Generalized phase-matching conditions for high harmonics: The role of field-gradient forces, Phys. Rev. A 55, 3204 (1997).

[45] M. B. Gaarde, J. L. Tate, and K. J. Schafer, Macroscopic aspects of attosecond pulse generation, J. Phys. B 41, 132001 (2008).

[46] A. D. Shiner, C. Trallero-Herrero, N. Kajumba, H.-C. Bandulet, D. Comtois, F. Légaré, M. Giguère, J.-C. Kieffer, P. B. Corkum, and D. M. Villeneuve, Wavelength Scaling of High Harmonic Generation Efficiency, Phys. Rev. Lett. 103, 073902 (2009).

[47] B. E. Schmidt, A. D. Shiner, M. Giguère, P. Lassonde, C. A. Trallero-Herrero, J.-C. Kieffer, P. B. Corkum, D. M. Villeneuve, and F. Légaré, High harmonic generation with long-wavelength few-cycle laser pulses, J. Phys. B 45, 074008 (2012). 